\title{
A joint high-resolution processing method and its application for thin inter-beds
}

\author{
Liu Zhiwei ${ }^{1,2 *}$ and Wang Yanchun ${ }^{1,2}$ \\ ${ }^{1}$ School of Geophysics and Information Technology, China University of Geosciences, Beijing 100083, China \\ ${ }^{2}$ Key Laboratory of Geo-detection, Ministry of Education, China University of Geosciences, Beijing 100083, China \\ (C) China University of Petroleum (Beijing) and Springer-Verlag Berlin Heidelberg 2013
}

\begin{abstract}
Seismic processing characterizing thickness and borders of thin inter-beds has gradually evolved from post-stack migration to pre-stack migration, and the latter considers both vertical and lateral resolutions. As the key processing methods for improving vertical and lateral resolution, conventional deconvolution and pre-stack time migration (PSTM) are not simply dominated by the estimation and compression of the wavelet because of its instability. Therefore, considering the variations of wavelet frequency before, during and after PSTM can obtain good common reflection point (CRP) gathers and imaging profiles of thin inter-beds. Based on the frequency characteristics of the wavelet before, during and after PSTM, a joint high-resolution processing method for thin inter-beds is proposed in this paper, including inverse $Q$ filtering for high-frequency compensation before PSTM, optimum weighting Kirchhoff PSTM for preserving high-frequencies during PSTM, and wavelet harmonizer deconvolution for consistent processing and frequency-band broadening after PSTM. An application to real data characterized by mudstone beds in the Oriente Basin proved that the joint high-resolution processing method is effective for determining the thickness and borders of thin inter-beds and is favorable for subsequent reservoir prediction and seismic inversions.
\end{abstract}

Key words: Thin inter-bed, seismic wavelet, inverse $Q$ filtering, optimum weight function, harmonizer deconvolution

\section{Introduction}

Generally, thin inter-beds refer to a geologic body which is composed of two or more thin layers intermingled with beds of more normal thickness. The lithology difference results in different wave velocities in neighboring layers, so the bottom and top interfaces of one layer have opposite reflection coefficients, and the travel time is short. Because of limited frequency band of the wavelet, the seismic reflection of thin inter-beds is a result of superposition and interference of seismic wavelets (Widess, 1973). Vertically, the superposition and interference weaken the reflectivity coefficients of interfaces, and decrease the resolution of thin inter-beds. A seismic interpreter cannot properly distinguish the boundaries of a single layer (Knapp, 1990). Laterally, some wedges exist in the positions with lithologic changes in the seismic profiles, even the mudstone beds. These strong events appear discontinuous because the diffracted amplitudes of two sides of the diffracted point cannot be weakened after migration for different velocities (Robertson and Nogami, 1984). Therefore, thin inter-beds imaging is complex and requires high vertical and lateral resolution.

*Corresponding author. email: zwliu007@sina.com

Received September 18, 2012
Seismic vertical resolution refers to the ability to identify reflected interfaces from wavelets. Rayleigh studied Fraunhofer optical diffraction and proposed that two neighboring peaks can be visually distinguished if the time interval of the wavelet is not less than the peak-trough interval $\Delta \tau_{R}$, and the resolution limit is a quarter of the wavelength, commonly called the Rayleigh criterion (Fig. 1). Ricker $(1945 ; 1953)$ studied the composite waveform by convolving a zero-phase wavelet with two pulses of equal amplitude and polarity, and showed that the composite waveform becomes flat between the two pulses when the time interval of the two pulses is $2 \Delta \tau_{r}$, which is defined as the vertical resolution limit, i.e., 1/4.6 of wavelength, now called the Ricker criterion (Fig. 1). Widess $(1973 ;$ 1982) studied a composite waveform by convolving a zero-phase wavelet with two pulses of equal amplitude and opposite polarity. He concluded that the composite waveform converges to the derivative of the wavelet with a decreasing interval between the two pulses and indicated that the resolution limit is $1 / 8$ of the wavelength if the amplitude of the composite wave is considered. Kallweit and Wood (1982) made a summary of vertical resolution and showed that the Ricker criterion can be applied to both equal and opposite polarity situations of one doublet. Van Riel and Berkhout (1985) pointed the disadvantages of the above criteria: 1) only the interference 
of the central peak with the first trough is considered, but the influence of side lobes of the wavelet on resolution is ignored. 2) Rayleigh and Ricker criteria are both based on an isolated doublet, but the multi-layer case is more important in practice. 3) A good result is obtained in the equal polarity situation, but in the opposite polarity situation, which is more important in practice, the result is not analyzed in detail. As a result, Berkhout introduced the wavelet length criterion for the vertical resolution. He proved that the dominant frequency is a key parameter in the Rayleigh and Ricker criteria. Knapp (1991) proposed the absolute resolution and concluded that the vertical resolution is related with the frequency of the wavelet, that is, the higher the frequency, the higher the resolution. The vertical resolution of seismic data can be improved in two ways, deconvolution and parameter inversion. Most parameter inversion methods assume that the formation is composed of a number of strong reflectors within a given depth range and the reflections of thin interbeds are considered as band-limited noise and are even ignored, so the method is not suitable for improving thin inter-bed imaging. Deconvolution assumes that any reflection is a convolution of the zero-phase wavelet with reflectivity coefficients. Deconvolution can obtain a series of smooth reflectivity coefficients and the key is wavelet compression and reshaping. As a result, the frequency bandwidth and the frequency of seismic wavelet play an important role in improving the vertical resolution of thin inter-beds.

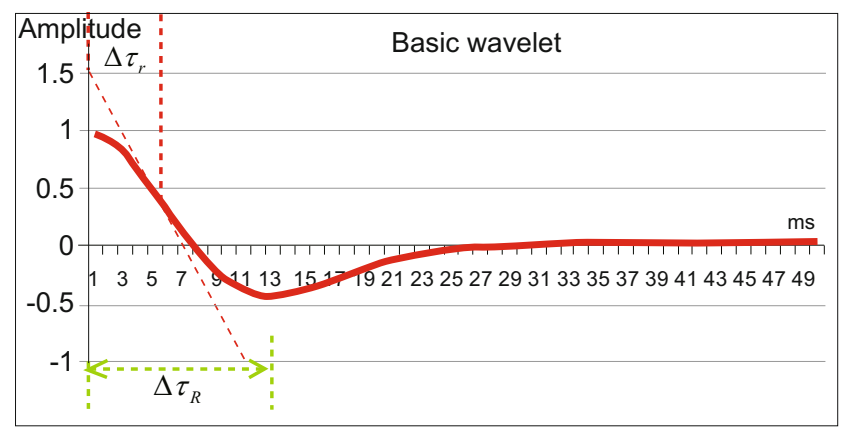

Fig. 1 Resolution limit of the zero-phase Ricker wavelet

The lateral resolution has been discussed by many authors on post-stack and pre-stack migration and diffraction tomography (Wu and Toksöz, 1987). Safar (1985) analyzed the limiting factors to the lateral resolution by Kirchhoff migration and concluded that seismic lateral resolution is controlled not only by the migration aperture and frequency but also by the weighting function, velocity accuracy and spatial sampling. Von Seggern (1994) proposed visible resolution for 3D geologic bodies. Knapp (1991) defined the Fresnel zone for lateral resolution study based on the relation of the Fresnel zone and wavelet length. Brühl and Vermeer (1996) pointed out that Knapp's definition in a narrow-band situation cannot converge to the classical Fresnel zone and gave a redefinition based on the radius of the reflector with maximum reflected energy. Vermeer (1998) analyzed factors affecting seismic lateral resolution, including the velocity model, migration aperture, fold and spatial sampling. Ma (2005) and Di and Gu (2005) qualitatively and quantitatively investigated seismic imaging resolution using geophysical models. Generally, the lateral resolution is evaluated by the Fresnel zone. Considering a flat reflector and a point source, the reflected energy of receiver is mostly from the interference of the first-order Fresnel zone. The energy from the higherorder Fresnel zones cancel each other, that is, the contribution of the first-order Fresnel zone determines the actual reflection response, as shown in Fig. 2. According to the Rayleigh criterion, the dominant frequency of a seismic wavelet determines the lateral resolution. In migration imaging, with the downward continuation of the source and receiver, the seismic wavefield increasingly approximates the actual reflector, and the Fresnel zone becomes smaller. Undoubtedly, migration is an essential approach to improve seismic lateral resolution. Pre-stack migration gradually replaced post-stack migration given the advantage of enhancing velocity analysis accuracy and preserving relative amplitudes. Accordingly, the improvement of lateral resolution of thin inter-beds relies on the dominant frequency of the seismic wavelet and pre-stack migration method.

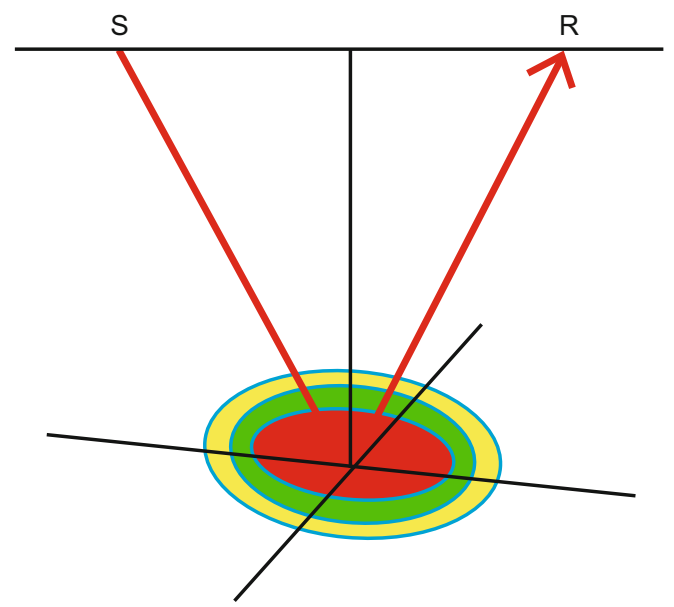

Fig. 2 Reflection interference of Fresnel zones

Therefore, the imaging processing of thin inter-beds should consider the frequency variation of wavelet before, during and after migration. Conventional surface consistent deconvolution (SCDC) and Kirchhoff pre-stack time migration (PSTM) have been proved to improve resolution for structural processing. However, for thin inter-beds, some new problems appear such as wavelet instability, amplitudepreservation of the weight function of Kirchhoff PSTM, and relative amplitude-preserved frequency expansion. We present a joint high-resolution processing method for thin inter-beds, emphasizing analyzing the frequency variations of wavelet before, during and after PSTM, and select corresponding techniques to improve vertical and lateral resolution, which are wavelet high frequency compensation before PSTM, wavelet frequency protection during PSTM and wavelet frequency consistent extension after PSTM.

\section{Model \& method}

\subsection{Inverse $Q$ filtering}

When a seismic wavefield propagates in thin inter-beds, high frequencies are lost because of spherical dispersion and 
stratigraphic filtering, so the seismic resolution reduces and signal instability enhances in later time (Deng, 1992; Varela et al, 1993; Mateeva, 2003). Fig. 3(a) shows that the seismic wavelet retains the frequency characteristics of a vibrating source in homogeneous media, whereas the wavelet's dominant frequency and bandwidth significantly decreases in a thin layer medium with linearly varying velocity, as shown in Fig. 3(b).

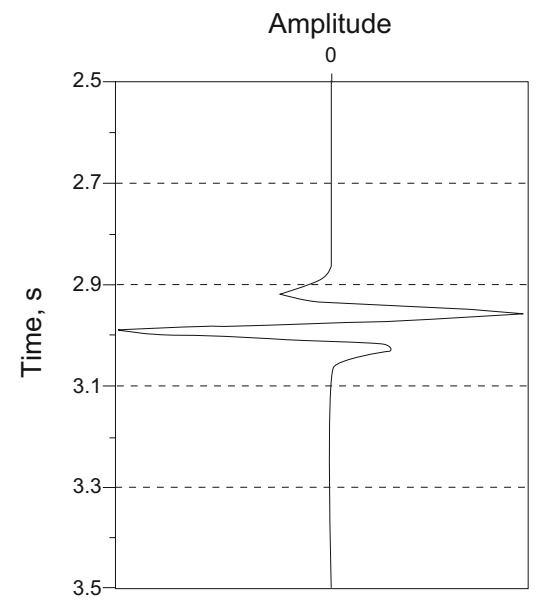

(a)

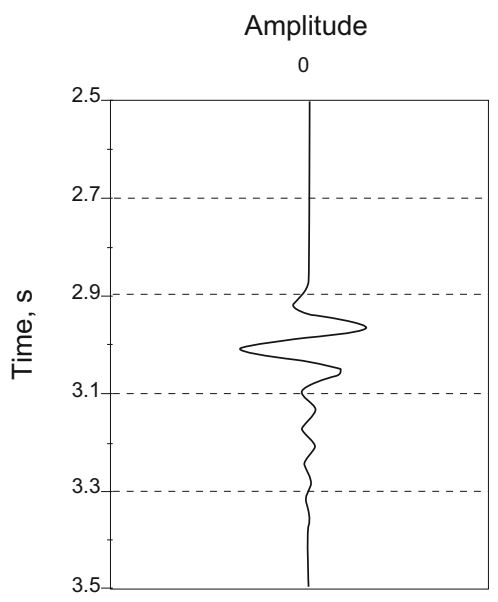

(b)

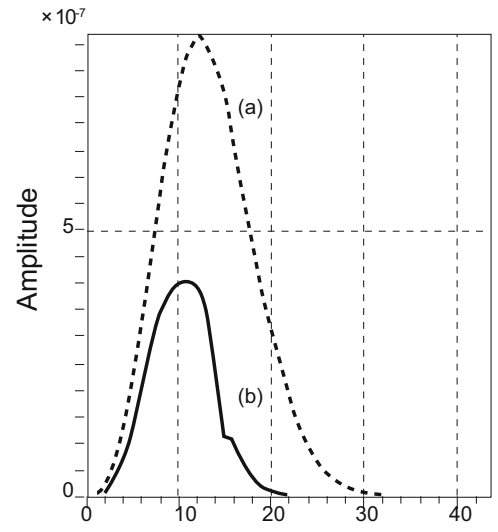

(c)

Fig. 3 High-frequency absorption of a seismic wavelet

(a) Homogenous medium, (b) Layer medium with linear velocities, (c) Spectra of (a) and (b)

Deconvolution, spectral whitening and frequency compensation based on wavelet transform are widely used in seismic processing (Gao et al, 2003). Constructing a timedomain Wiener optimal filter in deconvolution has to provide treatments for both early and late time seismic records, so the present deconvolution methods mainly have a windowdependent design. However, the design leads to destruction of seismic wavelets in the overlapping segments. Spectral whitening flattens the amplitude spectra as a kind of zerophase deconvolution, and when the reflection coefficient is not white noise, spectral whitening will damage the reflection coefficients. High-frequency compensation based on wavelet transform decomposes seismic data into different scales and then operates gain processing. Because the wavelet function and scale function at any time are orthogonal, the modeled processing is disadvantageous for the orthogonality. Wavelet stability and white reflection coefficients play a key role in the compensation of high frequency.

A seismic signal's instability results from inelasticity and inhomogeneity of subsurface media, and inverse spherical divergence and inelastic attenuation correction before deconvolution are performed. The high frequency attenuation can be described as a function of the quality factor $Q$ and be compensated by inverse $Q$ filtering (Goupillaud, 1961; Wang, 2002). During propagating, the seismic signal suffers phase distortion related to the frequency $f$ and quality factor $Q$, and the seismic signal can be written as

$$
W_{Q}(t, \tau, z)=\operatorname{IFT}\left\{W(f, \tau, z) \mathrm{e}^{-\pi \tau / Q[|f|+i H(|f|)]}\right\}
$$

Assuming that $Q$ does not vary with frequency, then

$$
W_{Q}(t, \tau, z)=\operatorname{IFT}\left\{W(f, \tau, z) \mathrm{e}^{-\pi f z /[Q v(f)]}\right\}
$$

where, $v(f)=v\left(f_{\mathrm{c}}\right)\left\{1+1 / \pi Q \ln \left|f / f_{\mathrm{c}}\right|\right\}$ and $f_{\mathrm{c}}$ is a cut frequency, and the phase velocity is determined by the wavelet frequency. Frequency attenuation increases with an increase in frequency and propagation time. For the solution of the $Q$ matrix, the most stable methods are discrete $Q$ in vertical seismic profile (VSP) and scanning $Q$ analysis in seismic data. Inverse $Q$ filtering can be used to compensate frequency-dependent seismic energy attenuation and phase distortion, and to improve the stability of the seismic signal (Kjartansson, 1979). We simulated inverse $Q$ filtering with the following results. Assuming that the constant $Q$ equals to 150, the wavelet is trace 1 in Fig. 4. The results of applying inverse $Q$ filtering to trace 1 with different $Q$ values are traces 2, 3, and 4 in Fig. 4, showing that when the $Q$ value is close to 150 , the result is the best.



Fig. 4 Simulation of inverse $Q$ filtering with different $Q$ values 


\subsection{Optimum weighting Kirchhoff PSTM}

Kirchhoff PSTM is based on the Kirchhoff integral solution of the wave equation, and its migration operator response is better than that of frequency-wavenumber (FK) and finite difference (FD) migrations (Wu and Toksöz, 1987). We used the inter-beds model as shown in Fig. 5(a) to simulate the seismic data with an FD acoustic wave equation. The width of the mudstone bed is $40 \mathrm{~m}$, and the wavelet is a Ricker wavelet with a dominant frequency of $35 \mathrm{~Hz}$. Then, the FK migration, FD migration, and Kirchhoff PSTM were performed, and the results were shown in Fig. 5(b), (c), and (d). We can see that the Kirchhoff migration has a great advantage in improving the vertical and lateral resolution. Even though the Kirchhoff PSTM improves the imaging accuracy of inter-beds, the problems of migration noise and amplitude preservation still exist which can affect the lateral resolution of thin inter-beds (Chen and Schuster, 1999). The weighting factor in the Kirchhoff PSTM operator directly influences the reflected wave amplitudes and frequency, so the Kirchhoff PSTM of thin inter-beds needs much consideration about the weighting factor.

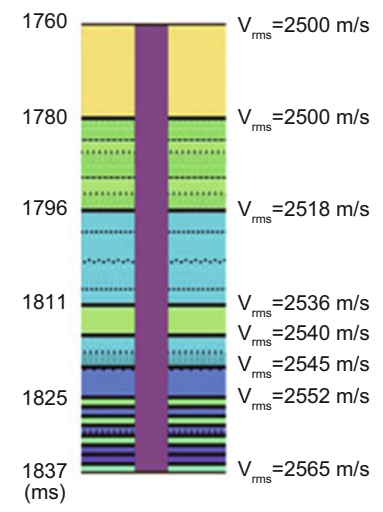

(a)

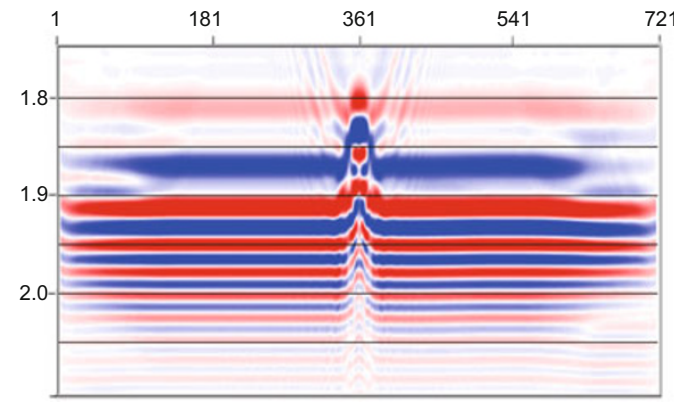

(b)

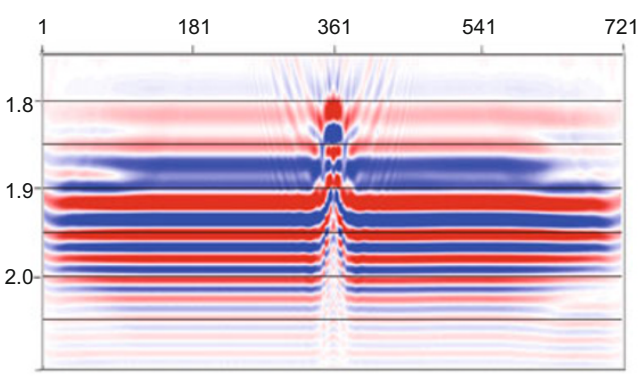

(c)

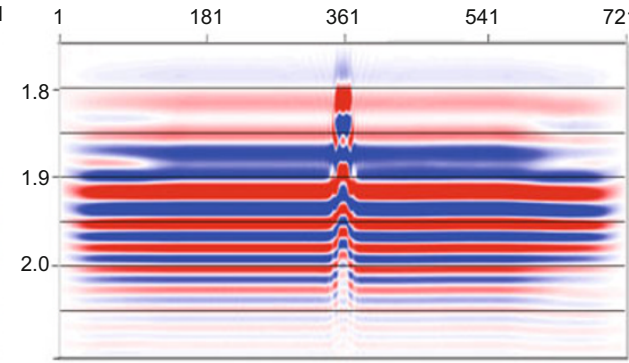

(d)

Fig. 5 Comparison of different migration methods for a thin inter-beds model

(a) Time model of thin inter-beds, (b) FK migration, (c) FD migration, (d) Kirchhoff migration

The Kirchhoff integral solution of the wave equation can be expressed as,

$P(x, y, z, \tau)=\frac{1}{4 \pi} \oint_{A}\left\{\left[\frac{\partial P}{\partial z}\right]+\frac{\cos \theta}{r^{2}}[P]+\frac{\cos \theta}{v r}\left[\frac{\partial P}{\partial t}\right]\right\} \mathrm{d} A$

where $[P]$ is the integral of the seismic wavefield in the range $A$ at $\tau=t-r / v$. The first term $[\partial P / \partial z]$ depends on the vertical variation of the seismic wavefield, and the second term is generally called the near-field source and mainly varies with $1 / r^{2}$. The two terms in computation are often ignored. The third term is generally called the far-field source and constitutes the basis of Kirchhoff migration. So Eq. (3) can be discretized as follows:

$$
\begin{aligned}
& P_{\text {out }}\left(x_{\text {out }}, y_{\text {out }}, z, \tau=2 z / v\right) \\
& =\frac{\Delta x \Delta y}{4 \pi} \sum \frac{\cos \theta}{v r} \frac{\partial}{\partial t} P_{\text {in }}\left(x_{\text {in }}, y_{\text {in }}, z=0, \tau=t-r / v\right)
\end{aligned}
$$

where, $\Delta x$ and $\Delta y$ are horizontal and vertical trace intervals, and $\cos \theta$ is the dip factor or direction factor, and $1 / v r$ is the spherical spreading factor used for the corrections of amplitudes and phases. Eq. (4) implies that a reflector can be imaged at the positions with maximum amplitudes derived from the summations of seismic wavelets across the propagating path from source to receiver. However, the summations lead to a high-frequency reduction in Kirchhoff pre-stack migration (Tygel et al, 1994). Theoretically, the amplitude of any imaging point in the bin should equal to the stacked value of different offset tracks in the bin. The final imaging point lies in the point of tangency with the maximum amplitude and the amplitudes of other points are weakened. Practically, the high-frequency attenuation causes an increase of seismic wavelet duration and a decrease of vertical and lateral resolution. The diffraction stack principle is shown in Fig. 6. 


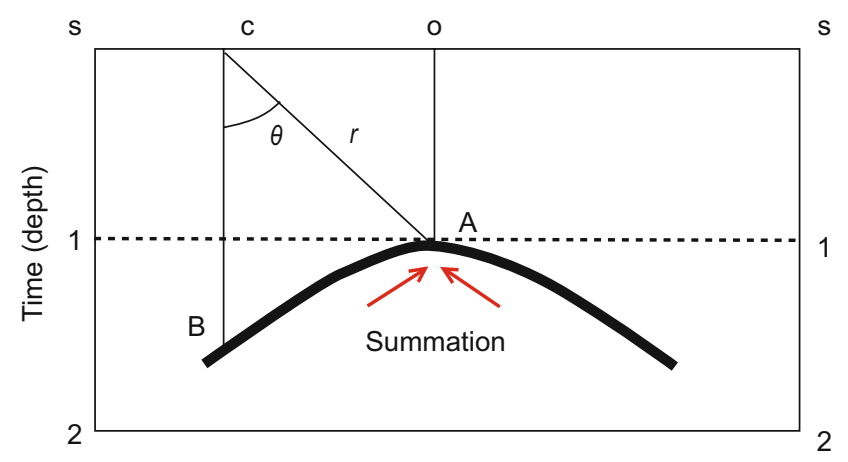

Fig. 6 Diffraction stack in Kirchhoff migration

The influence of Kirchhoff pre-stack migration on wavelet frequency mainly focuses on the weighting factors which include the wavefront spreading factor, dip factor (direction factor) and wavelet shaping factor (Bancroft, 2007). The wavefront spreading factor represents the amplitude attenuation when the wavelet propagates, and conventional preprocessing usually performs spherical spreading compensation. Therefore, this factor does not need to be applied in Kirchhoff migration. The dip factor (direction factor) has a maximum value at the apex of diffraction and has the largest contribution to integral summation. However, most cases proved that the aperture in Kirchhoff migration is more important to imaging than the dip factor. Reflection imaging and diffraction convergence rely on diffraction stacking. If we assume a band-limited wavelet, the reflected wave is identical to the left side of the diffracted wave in phase, whereas it has a $180^{\circ}$ phase difference from the right side of the diffracted wave. Then, whether or not to use a wavelet shaping factor in Kirchhoff PSTM depends on the contrast between the migrated trace and corresponding welllog curves (Qian, 2008). In summary, Kirchhoff PSTM for inter-beds imaging should eliminate the effect of spherical spreading factor, enhancing migration aperture, and determining the wavelet shaping factor from well-log curves.

\subsection{Wavelet harmonizer deconvolution}

Deconvolution is one of the standard processing steps. The basic assumptions of deconvolution is minimum phase, invariance of seismic wavelet and white reflection coefficients, and the optimum Wiener filter based on minimum square norm can be designed for improving seismic vertical resolution. In addition, some nonlinear filters are also designed based on other norms (Schoenberger, 1974; Okaya, 1995; Marfurt and Kirlin, 2001). Applications in practice have verified that a single-trace deconvolution algorithm cannot adapt to relative amplitude-preserved lithologic processing of inter-beds, and multi-trace processing should be used. For example, surface consistent deconvolution (SCDC) and multi-trace predictive deconvolution can basically achieve a vertical and lateral wavelet consistency and improve seismic resolution before CMP/CRP stack (Robinson, 1967; Peacock and Treitel, 1969; Li and Guo, 2007). Even if considering the optimal weighting factor, diffraction convergence in the Kirchhoff PSTM will lead to a decrease of frequency (Bancroft, 2007), as shown in Fig. 7. Moreover, the temporal and spatial inconsistency of wavelet in preprocessing will lead to mixing of frequencies, so as to influence the thin interbeds imaging to a certain degree.

Therefore, wavelet consistent processing is necessary after Kirchhoff PSTM. After preprocessing deconvolution and PSTM, the wavelets of different offsets and depths in CRP gathers are consistent, so single-trace predictive deconvolution is used for further improving resolution. Conventional single-trace deconvolution based on the assumption of a minimum-phase wavelet generally obtains the deconvolution operator from different time windows and leads to amplitude distortion in the boundaries of time windows (Huang et al, 2007; Clarke, 1968), as shown in Fig. 8. In harmonizer deconvolution, the operator length and predictive distance are determined first, and then the deconvolution operator is calculated for every sampling point of the seismic trace, as shown in Fig. 8. The calculated deconvolution operators may vary in time and space, which

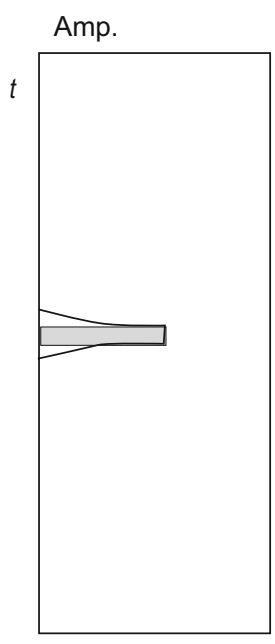

(a)

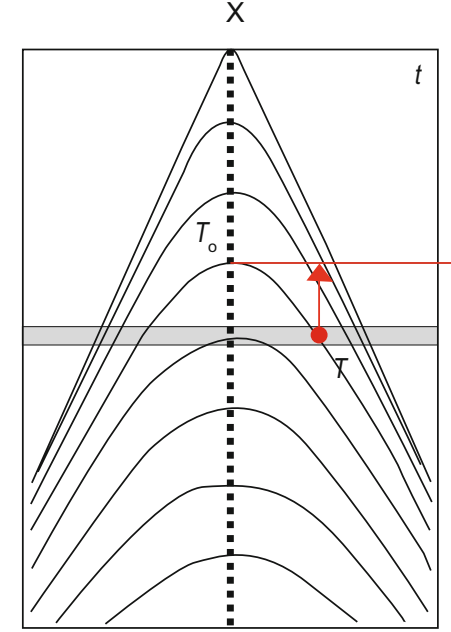

(b)

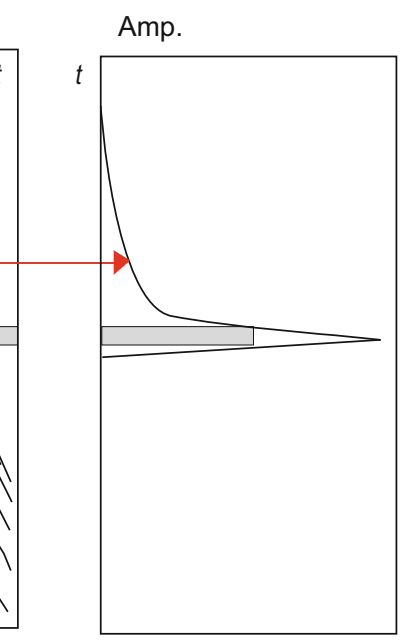

(c)

Fig. 7 The effect of Kirchhoff migration on a seismic wavelet

(a) Wavelet before migration, (b) Kirchhoff diffraction stack path, (c) Wavelet after migration 


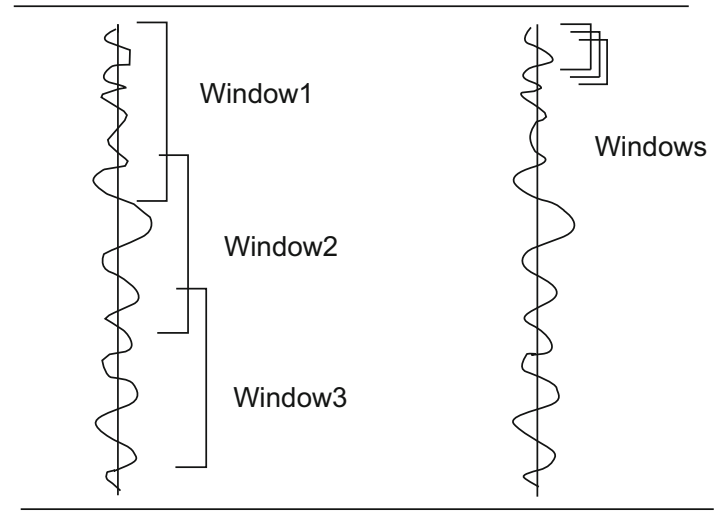

Fig. 8 Time windows of conventional (left) and harmonizer (right) deconvolution

can be regarded as a correction of wavelet inconsistency.

\section{Application}

The Oriente Basin in Ecuador, South America, is a foreland basin, with low-relief traps and sets of thin interbeds in the $\mathrm{X}$ block. The objective layer $\mathrm{Y}$ has a mudstone bed, whose origin is structural and sedimentary. The lithology difference has been verified by drilling. The surface conditions of seismic survey are plain, swamp and jungle, and a stable and horizontal refraction surface (refraction velocity is $2,000 \mathrm{~m} / \mathrm{s}$ ) exists. Apart from strong groundroll waves, seismic noise mainly consists of low and high frequency abnormal noise distributed irregularly. The valid frequency ranges from $8 \mathrm{~Hz}$ to $45 \mathrm{~Hz}$ in the $\mathrm{Y}$ layer, and the root mean square (RMS) velocity approximates to 3,000 $\mathrm{m} / \mathrm{s}$. The processing task is to investigate contacting relations of thin inter-beds and identifying the lateral boundaries of the mudstone bed.

In preprocessing, a zone filter is used for eliminating ground-roll waves and suppressing low-frequency abnormal amplitudes. Based on spherical spreading compensation, surface-consistent amplitude processing including surfaceconsistent amplitude compensation (SCAC), zone amplitude processing (ZAP), and surface-consistent deconvolution (SCDC) are applied for globally preserving horizontal changes of amplitudes. Along the Y layer, negative maximum amplitudes are extracted from the stacked volume after Kirchhoff PSTM, and the mudstone bed (in the white circle) appears unclear in Fig. 9(a). From Fig. 9(a), the horizontal boundary of the mudstone bed is not clear, abnormal amplitudes exist and the whole attribute is not balanced. We use inverse $Q$ filtering before SCDC, a similar amplitude attribute extracted from the new migration volume shows that the abnormal amplitudes are suppressed and the sand blocks and mudstone bed become clear in Fig. 9(b). Fig. 10 shows the results of corresponding stack section and time-frequency spectra. We can see that inverse $Q$ filtering can efficiently compensate high frequency information and improve the vertical and lateral resolution of thin inter-beds.

In Fig. 9(b), there are some abnormal sand blocks and the lateral resolution near the mudstone bed is low. According to

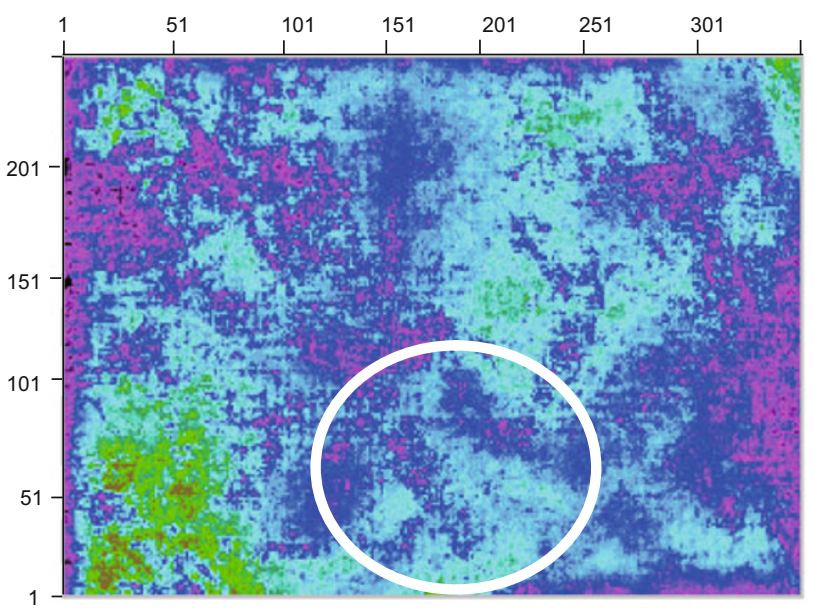

(a)

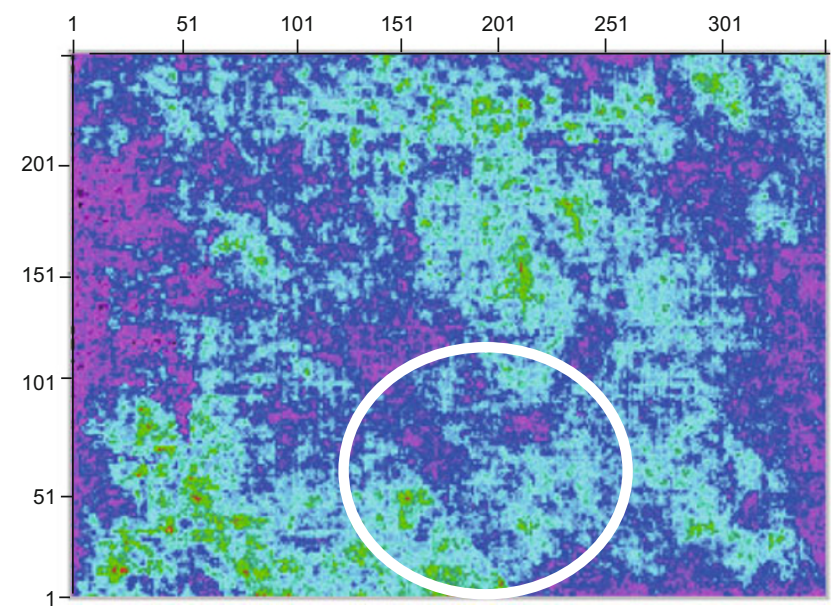

(b)

Fig. 9 Maximum negative amplitudes extracted from the $\mathrm{Y}$ layer (Grid unit: $25 \mathrm{~m} \times 50 \mathrm{~m}$ ). (a) Before applying inverse $Q$ filter, (b) After applying inverse $Q$ filter

the above analysis of the Kirchhoff migration, we correct the weighting factors of the Kirchhoff PSTM, including inverting the spherical spreading compensation factor, decreasing the dip factor and adjusting the shaping factor with reference to the well-log. After PSTM, the amplitude attribute similar to Fig. 9 is extracted along layer $\mathrm{Y}$ as shown in Fig. 11 and the waveform of the Y layer is compared with the well$\log$ in Fig. 12. It is verified that the Kirchhoff PSTM for thin inter-beds does not have to correct the shaping factor, while the spherical spreading and dip factor play a key role in preserving amplitude attribute and improving the vertical resolution. Additionally, Fig. 12 also shows that the optimal weighting Kirchhoff migration protects the high-frequency and low-frequency reduction introduced by migration.

Although SCDC can be used to obtain lateral consistency of wavelets and eliminate parts of near subsurface influences on the wavelets, Fig. 14(a) shows that the vertical resolution of the inter-beds is not high enough to separate thin layers and the amplitude spectra is still flat. In order to separate 

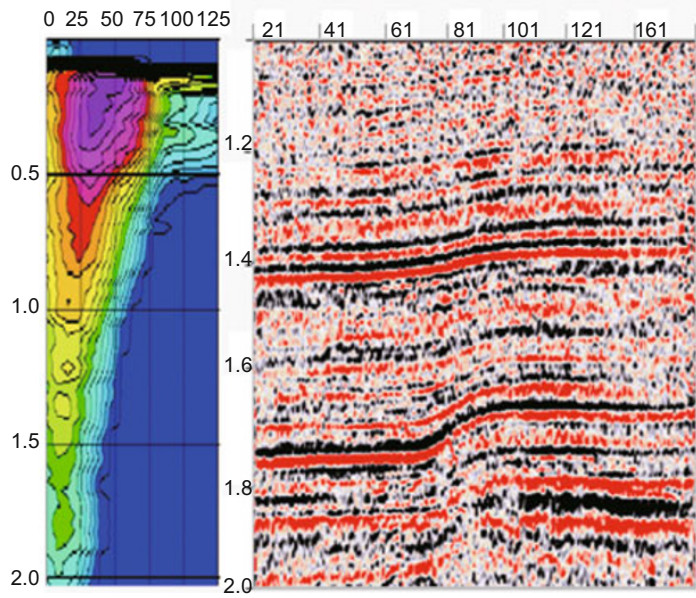

(a)

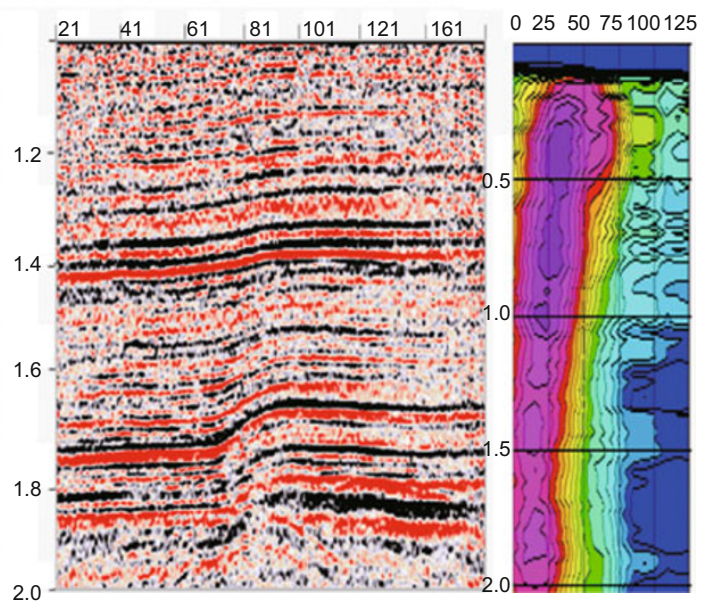

(b)

Fig. 10 Time-frequency spectra and sections of Inline No.50. (a) Before applying inverse $Q$ filter, (b) After applying inverse $Q$ filter

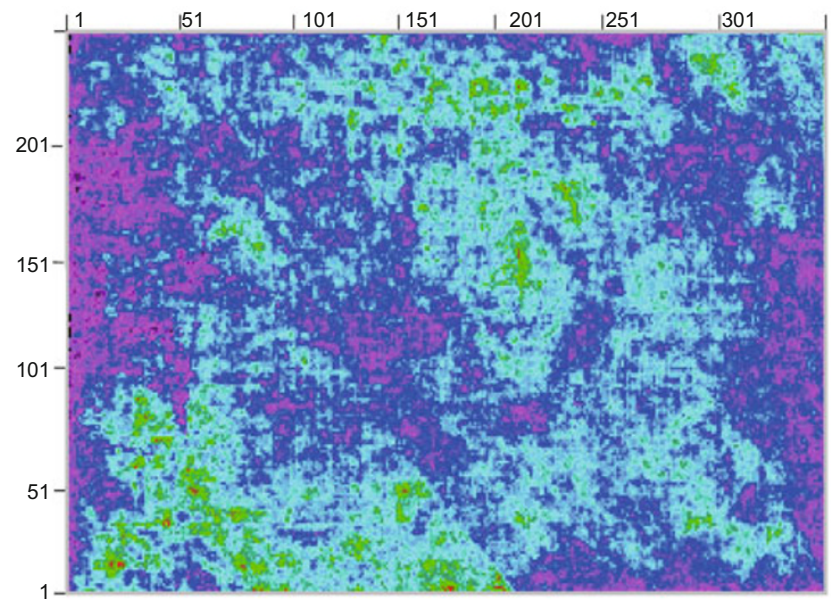

(a)

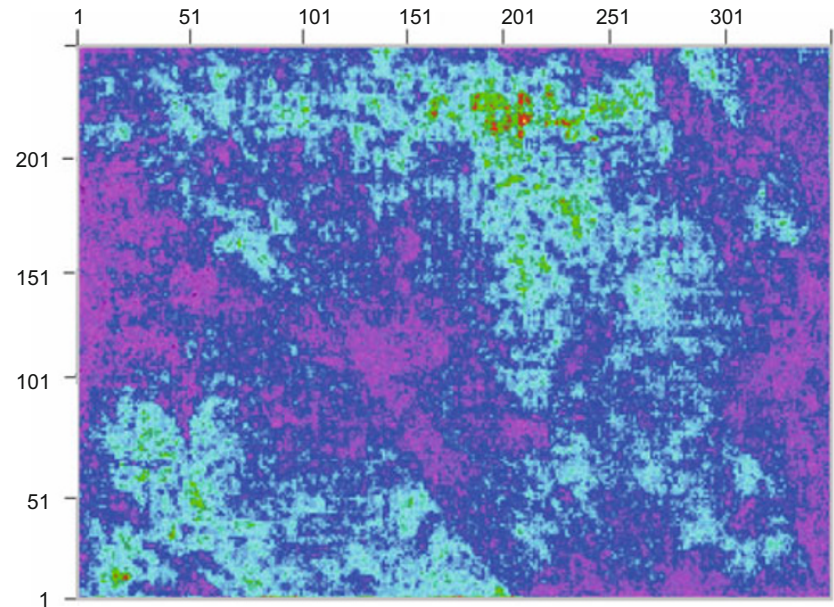

(b)

Fig. 11 Maximum negative amplitudes extracted from the $Y$ layer (Grid unit: $25 \mathrm{~m} \times 50 \mathrm{~m}$ ) (a) Conventional Kirchhoff migration, (b) Optimal weighting Kirchhoff migration

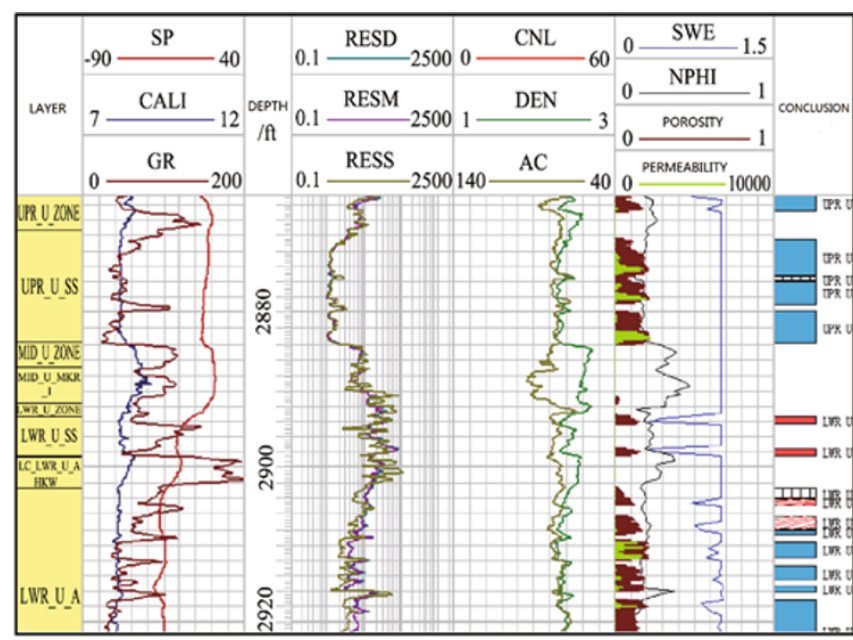

(a)



(b)

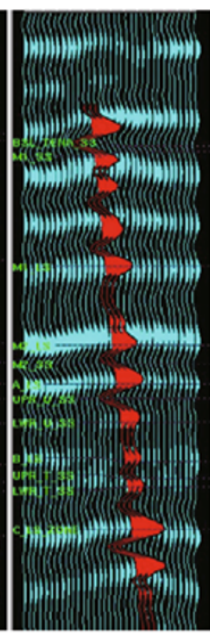

(c)

Fig. 12 (a) Well No.1's log, (b) Traces without optimal Kirchhoff PSTM, (c) Traces after optimal Kirchhoff PSTM 
thin inter-beds, we must improve the vertical resolution of CRP gathers. Based on the fixed wavelet operator length and predictive distance, the wavelet harmonizer deconvolution designs a filter varying with time and space on CRP gathers for broadening bandwidth. The attribute of negative maximum amplitude is shown in Fig. 13(b). A large mudstone bed looks very clear and some small and narrow beds also appear in the left bottom of the attribute map. Fig. 14 shows the results of stack section and spectra before and after applying wavelet harmonizer deconvolution. It is noted that thin inter-beds are



(a) distinguished and frequency spectra are also flattened. Fig. 15 shows the comparison with well-log before and after applying wavelet harmonizer deconvolution.

Finally, the resolution of the $\mathrm{X}$ block is increased by the joint method, and the thin inter-beds and boundaries of the mudstone bed can be identified clearly. Additionally, some small-scale geologic formations are well imaged, like thinner inter-beds and low-relief traps. The final profiles and CRP gathers can meet the requirements of fine reservoir prediction and seismic inversion.

Fig. 13 Maximum negative amplitudes extracted from the Y layer (Grid unit: $25 \mathrm{~m} \times 50 \mathrm{~m}$ ).

(a) Before wavelet harmonizer deconvolution, (b) After wavelet harmonizer deconvolution

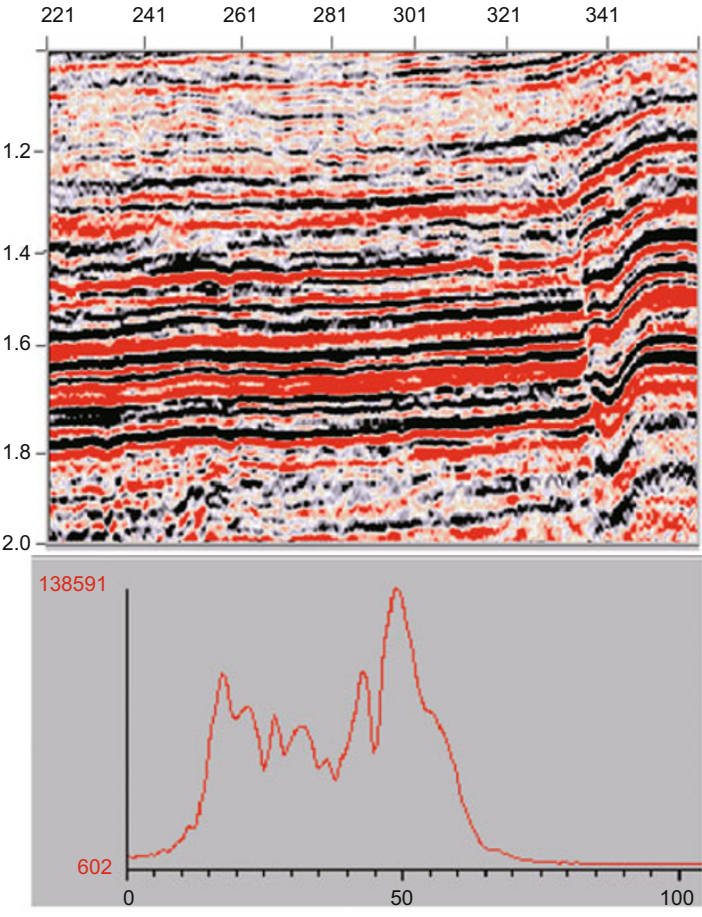

(a)


(b)

Fig. 14 Stacked sections and amplitude spectra before and after wavelet harmonizer deconvolution. (a) Before wavelet harmonizer deconvolution, (b) After wavelet harmonizer deconvolution 


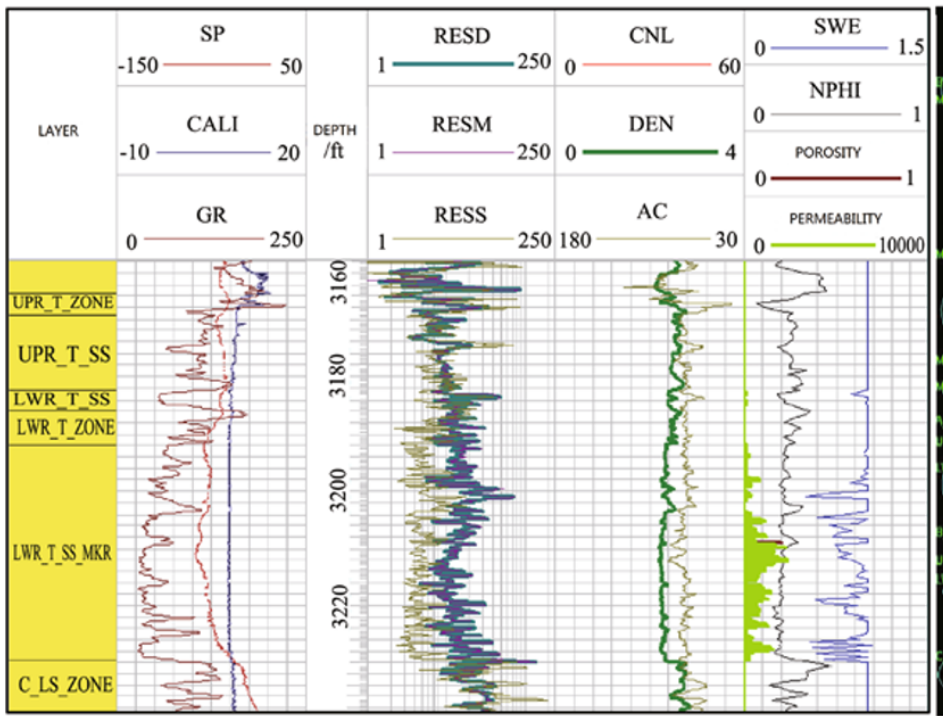

(a)



(b)

(c)

Fig. 15 (a) Well No.2's log, (b) Traces without wavelet harmonizer deconvolution, (c) Traces after wavelet harmonizer deconvolution

\section{Conclusions}

Thin inter-bed imaging is a completely high-resolution lithologic processing method and only joint high-resolution processing can accurately identify the thickness and borders of thin inter-beds. In addition, imaging of thin inter-beds is directly related to other steps of seismic processing and will fail if a wrong geometry definition, inaccurate static correction, inaccurate RMS velocity, and unsuitable frequency broadening methods are used. In this paper, the joint high-resolution processing method based on amplitude preservation can effectively increase the resolution of thin inter-beds. The key of joint high-resolution method for imaging thin inter-beds lies in the following aspects:

1) Applying inverse $Q$ filtering before SCDC can compensate for high-frequency loss and the amplitudes can reflect the lateral and vertical variations of subsurface lithology.

2) Optimal weighting Kirchhoff PSTM based on amplitude compensation, dip, and well-log data can retain the structural and lithologic characteristics of thin inter-beds and reduce frequency mixing.

3) Using wavelet harmonizer deconvolution on CRP gathers can globally unify the seismic wavelets and further enhance the vertical and lateral resolution of thin inter-beds.

\section{Acknowledgements}

We are very grateful to senior engineer Liu Jianhong, senior engineer Duan Wensheng and senior engineer Liu Junjie for their scientific advice and assistance during the study.

\section{References}

Bancroft J C. A Practical Understanding of Pre- and Post-stack Migrations (Volume 2). SEG. 2007

Brühl M, Vermeer G J O and Kiehn M. Fresnel zones for broadband data. Geophysics. 1996. 61(2): 600-604

Chen J and Schuster G T. Resolution limits of migrated images. Geophysics. 1999. 64(8): 1046-1053

Clarke G K C. Time-varying deconvolution filters. Geophysics. 1968. 33(6): 936-944

Deng H L. Seismic wave propagation in thinly-layered media with steep reflectors. Master Thesis. Center for Wave Phenomena, Colorado School of Mines. 1992

Di B R and Gu P C. Quantitative analysis of resolution of seismic migration imaging. Journal of the University of Petroleum. 2005. 29(5): 23-32 (in Chinese)

Gao J H, Chen W C, Li Y M, et al. Generalized S transform and seismic response analysis of thin interbeds. Chinese Journal of Geophysics. 2003. 46(4): 526-532 (in Chinese)

Goupillaud P L. An approach to inverse filtering of near-surface layer effects from seismic records. Geophysics. 1961. 26(4): 754-760

Huang J B, Gao L J and Gao Y. Side lobes of wavelets impact identification of thin sand bodies. Applied Geophysics. 2007. 4(2): $111-117$

Kallweit R S and Wood L C. The limits of resolution of zero-phase wavelets. Geophysics. 1982. 47(7): 1035-1046

Kjartansson E. Constant Q-wave propagation and attenuation. Journal of Geophysical Research. 1979. 82(1): 4737-4748

Knapp R W. Fresnel zones in the light of broadband data. Geophysics. 1991. 56(3): 354-359

Knapp R W. Vertical resolution of thick beds, thin beds, and thin-bed cyclothems. Geophysics. 1990. 55(9): 1183-1190

Li Z S and Guo X B. Predicting the distribution of thin bed reservoirs by broad frequency band seismic. Applied Geophysics. 2007. 4(2): 118126

Marfurt K J and Kirlin R L. Narrow-band spectral analysis and thin-bed tuning. Geophysics. 2001. 66(4): 1274-1283

Mateeva A A. Thin horizontal layering as a stratigraphic filter in absorption estimation and seismic deconvolution. Ph.D. Thesis. Colorado School of Mines. 2003

Ma Z T. Theoretical analysis of reflection seismic imaging resolution. Journal of Tongji University (Natural Science). 2005. 33(9): 11441153 (in Chinese)

Okaya D A. Spectral properties of the earth's contribution to seismic resolution. Geophysics. 1995. 50(1): 241-251 
Peacock K L and Treitel S. Predictive deconvolution: Theory and practice. Geophysics. 1969. 34(1): 155-169

Qian R J. Characteristics of the Seismic Waves and Related Technical Analysis. Beijing: Petroleum Industry Press. 2008 (in Chinese)

Ricker N. The computation of output disturbances from amplifiers for true wavelet inputs. Geophysics. 1945. 10(4): 207-220

Ricker N. Wavelet contraction, wavelet expansion and the control of seismic resolution. Geophysics. 1953. 18(4): 769-792

Robertson J D and Nogami H H. Complex seismic trace analysis of thin beds. Geophysics. 1984. 49(4): 344-352

Robinson E A. Predictive decomposition of time series with application to seismic exploration. Geophysics. 1967. 32(3): 418-484

Safar M H. On the lateral resolution achieved by Kirchhoff migration. Geophysics. 1985. 50(8): 1091-1099

Schoenberger M. Resolution comparison of minimum-phase and zerophase signals. Geophysics. 1974. 39(6): 826-833

Tygel M, Schleicher J and Hubral P. Pulse distortion in depth migration. Geophysics. 1994. 59(10): 1561-1569
Van Riel P and Berkhout A J. Resolution in seismic trace inversion by parameter estimation. Geophysics. 1985. 50(9): 1440-1455

Varela C L, Rosa A L R and Ulrych T J. Modeling of attenuation and dispersion. Geophysics. 1993. 58(8): 1167-1173

Vermeer G J O. Factors affecting spatial resolution. The Leading Edge. 1998. 17(8): 1025-1030

Von Seggern D. Depth-imaging resolution of 3-D seismic recording patterns. Geophysics. 1994. 59(4): 564-576

Wang Y H. A stable and efficient approach of inverse Q filtering. Geophysics. 2002. 67(2): 657-663

Widess M B. How thin is a thin bed? Geophysics. 1973. 38(6): 11761180

Widess M B. Quantifying resolving power of seismic systems. Geophysics. 1982. 47(8): 1160-1173

Wu R S and Toksöz M N. Diffraction tomography and multisource holography applied to seismic imaging. Geophysics. 1987. 52(1): 1125

(Edited by Hao Jie) 